\title{
Dissemination of Nutritional Knowledge in Germany - Nutrition Circle, 3D Food Pyramid and 10 Nutrition Guidelines
}

\author{
Peter Stehle \\ Department of Nutrition and Food Sciences, University Bonn and DGE, Bonn, Germany
}

\section{Key Words}

Wholesome diet • Nutrition circle $\cdot 3 \mathrm{D}$ food pyramid . German Nutrition Society $\cdot$ Reference values

\begin{abstract}
Background/Aims: Presentation of the general concept of the German Nutrition Society for a successful dissemination of nutritional knowledge. Methods: Evaluation of actual material used for nutrition education and teaching. Results: In Germany, different validated instruments on the basis of actual scientific evidence are available. Quantitative aspects can be easily enlightened with the help of the Nutrition Circle. Information concerning optimal personal behavior (lifestyle) is transported using a flyer with 10 Nutrition Guidelines. The 3D Food Guide Pyramid is an encouraging new instrument to establish new teaching programs with a high level of acceptance. It is planned to establish target-specific food guide pyramids, e.g. for adolescents, aged persons (considering gender), people with increased physical activity and others. Tests with target groups (health professionals, teachers, young consumers) showed a high rate of acceptance and demonstrated successful application of the German concept of dissemination of dietary guidelines. Conclusion: Using modern communication technology, it is feasible for the consumers and the health professionals to download gender- and age-specific scientific information as well as validated tools for successful nutrition education.
\end{abstract}

Copyright $\odot 2007$ S. Karger AG, Basel

\section{KARGER}

Fax +4161306 1234

E-Mail karger@karger.ch

www.karger.com
C) 2007 S. Karger AG, Basel

0250-6807/07/0518-00021\$23.50/0

Accessible online at:

www.karger.com/anm

\section{Introduction}

Dissemination of nutritional knowledge is a valuable and widely accepted preventive measure to diminish the incidence and prevalence of chronic (non-communicable) diseases in a population. In Germany, the German Nutrition Society (Deutsche Gesellschaft für Ernährung, DGE) owns the task to establish and publish scientifically sound food-based guidelines for a balanced and wholesome diet.

In close cooperation with our sister societies in Austria and Switzerland, reference values for nutrient intake are first established [1]. These nutrient-based recommendations are then 'translated' into food-based dietary guidelines with the aim to inform the consumer how a wholesome diet should be composed as well as to teach health professionals working in the different fields of nutrition education.

The goal of the present review is to describe in detail the general procedure from nutrients to food and to present new educational tools for a successful dissemination of dietary guidelines.

\section{Quantitative Aspects - the Nutrition Circle}

According the so-called 'nutritive' approach (transfer of actual reference values for essential nutrients into food patterns) [2], the Nutrition Circle (fig. 1) illustrates the 
Fig. 1. The Nutrition Circle.

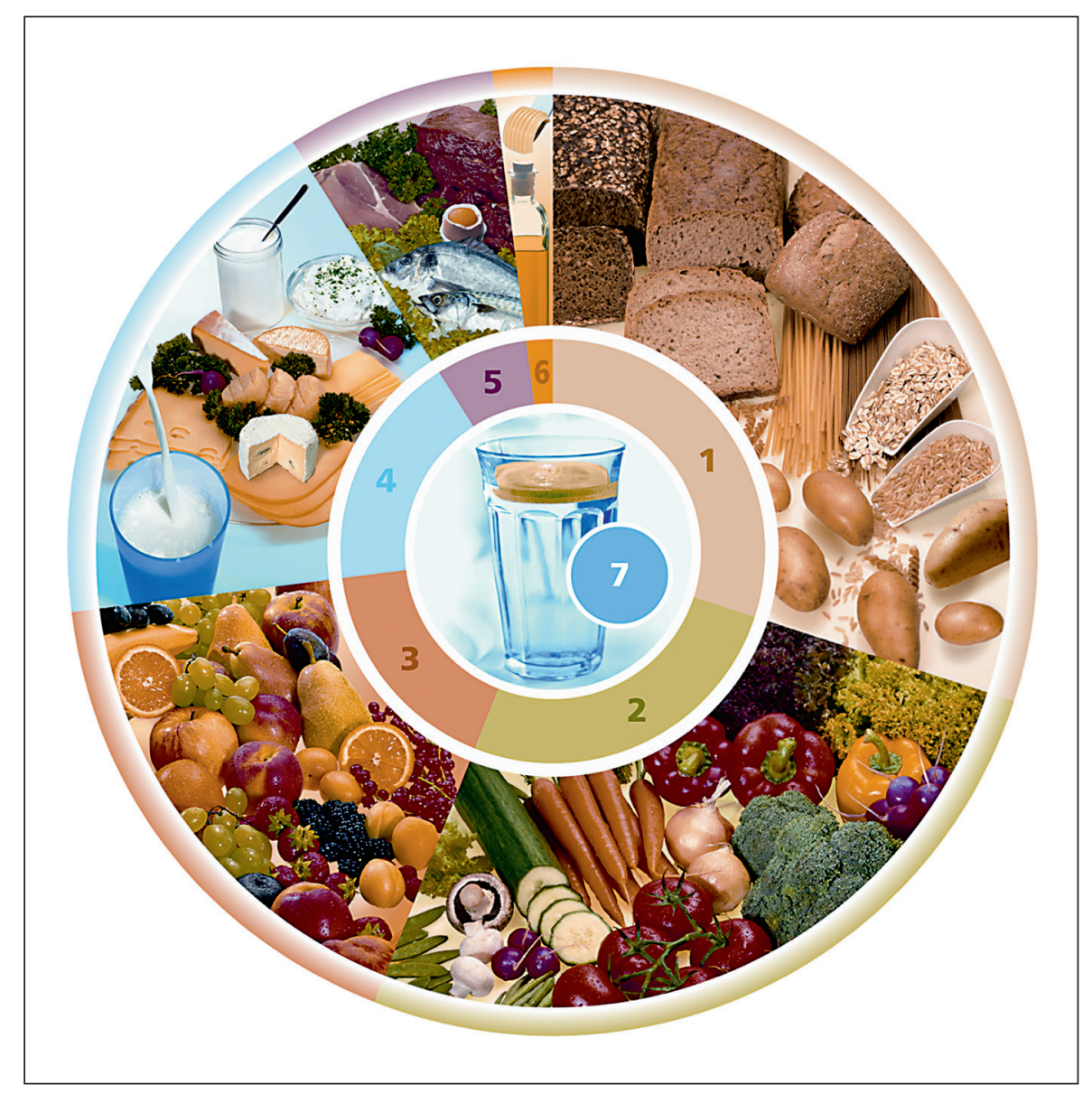

recommended daily intake in weight percent of 7 food classes (cereals/grains, vegetables, fruits, milk products, meat/fish, oils/fats, beverages) as calculated on the basis of energy, macro-, and micronutrient needs (quantitative aspects; visualized by the respective sector area except for beverages). Considering age- and sex-specific dietary allowances (calculations made for 4 groups: men and women aged $25-51$ and $>65$ years, respectively) and a physical activity level (PAL) of 1.4, exemplary daily plans using common foods are composed. The goal is to cover reference values for all nutrients when considering the daily intake averaged over 7 days. Food choice is strongly adjusted to the '5-a-day' principle (5 servings of fruit and vegetable per day) and the idea to use food with a high nutrient density (e.g., whole grain cereals). Food products with a known poor nutritional quality like sweets and alcoholic/energy-rich beverages are not considered.

The Nutrition Circle convincingly demonstrates that it is possible to provide almost all essential and important non-essential nutrients in adequate amounts by choosing high-quality food products available in supermarkets and traditional groceries. To cover iodine needs, $2 \mathrm{~g}$ of iodinated salt have to be consumed daily. The actual RDA for folate (folic acid) is difficult to reach even when folaterich vegetables are consumed regularly in high amounts. In line with political measures already taken for example in USA and Canada [3], the DGE is, thus, in favor to enrich flour often used in bakeries with synthetic folic acid $(150 \mu \mathrm{g} / 100 \mathrm{~g})$ [4] to improve average intake of folate/folic acid. The low vitamin D intake from food is counteracted by its endogenous synthesis in the skin catalyzed by UVB exposition. Following the advice given in the Nutrition Circle, the percentage of energy from fat ranged from 28 to $31 \%$; carbohydrates and protein provide $52-53 \%$ and $16-17 \%$, respectively.

Indeed, the Nutrition Circle only considers foods with a known high physiological quality and, thus, has to be seen as a target which should be reached by the consumer. 


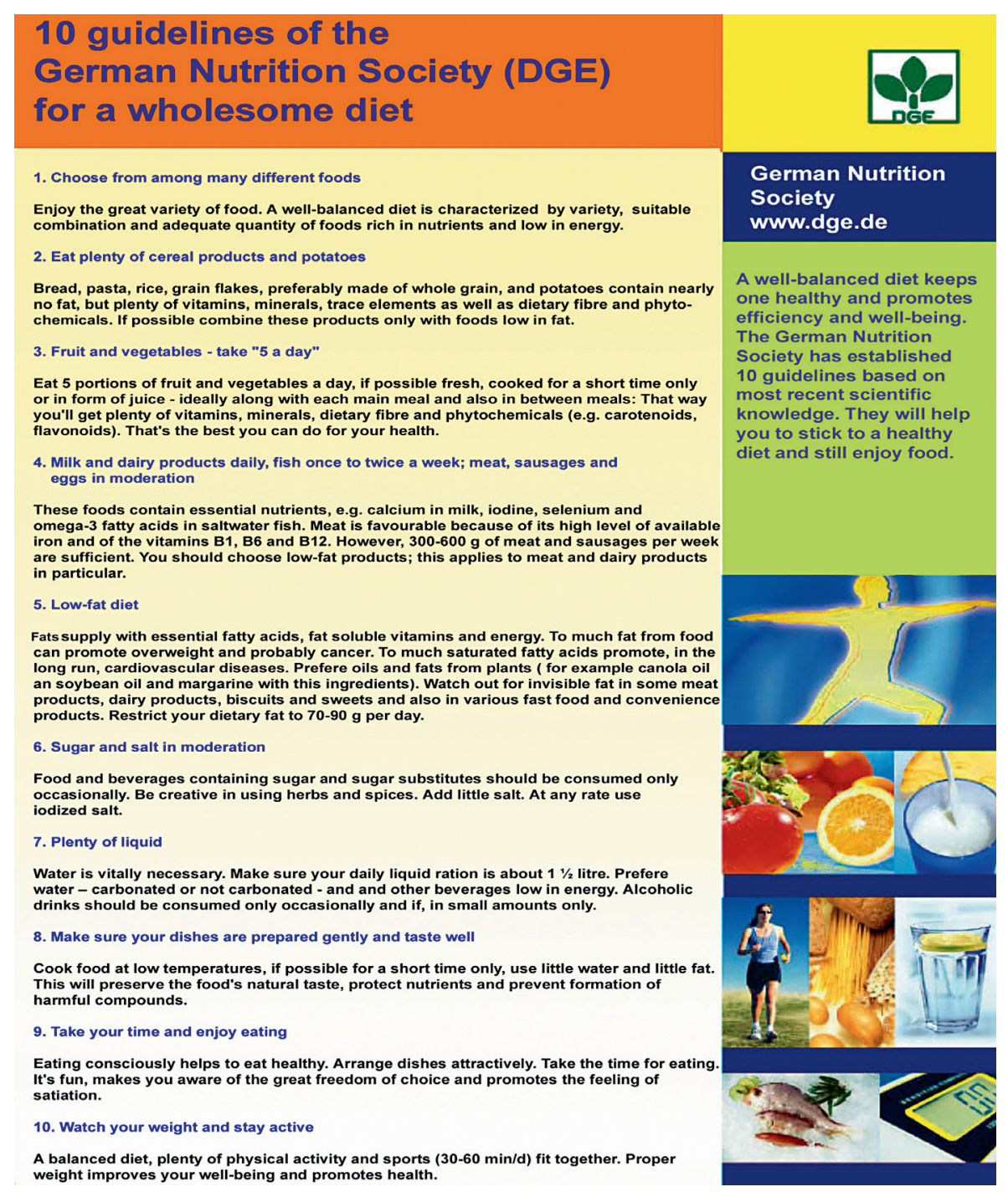

Fig. 2. The 10 Nutrition Guidelines.

A balanced diet, plenty of physical activity and sports (30-60 min/d) fit together. Proper
weight improves your well-being and promotes health.

German Nutrition Society

www.dge.de

A well-balanced diet keeps one healthy and promotes efficiency and well-being. Society has established 0 guidelines based on most recent scientific knowledge. They will help you to stick to a healthy t and still enjoy food.

\section{Physical, Social and Technological Aspects - 10 Nutrition Guidelines}

In addition to distinct nutritive aspects, the $10 \mathrm{Nutri-}$ tion Guidelines (fig. 2) consider important factors of lifestyle (e.g., eating out, wellness), physical activities (recommendation to regularly perform sports), and household (e.g., food processing, storage). This one-page flyer can be easily understood and brought into practice by the consumer. In contrast to the Nutrition Circle, the Nutrition Guidelines do not only focus on the 'optimal behavior' but give more 'real' advice to follow a wholesome diet and lifestyle in daily routine.

\section{Qualitative Aspects - the 3D Food Pyramid}

Graphs are mandatory educational tools for a successful dissemination of nutritional knowledge. Pyramids, circles and other geometric forms are frequently used to help consumers in realizing nutritional guidelines $[5,6]$. In recent years, the number of graphs published has increased tremendously, but unfortunately it is not always possible for the consumers to follow the rationale behind them. Presently available illustrations show obvious cons: provision of only quantitative (amount/portions of foods; see Nutrition Circle) or qualitative information (ranking of foods), space limitations, specific target groups (adolescents, young consumers) are not reached. Consequently, the DGE decided to establish a new graphic tool $(3 D$ 


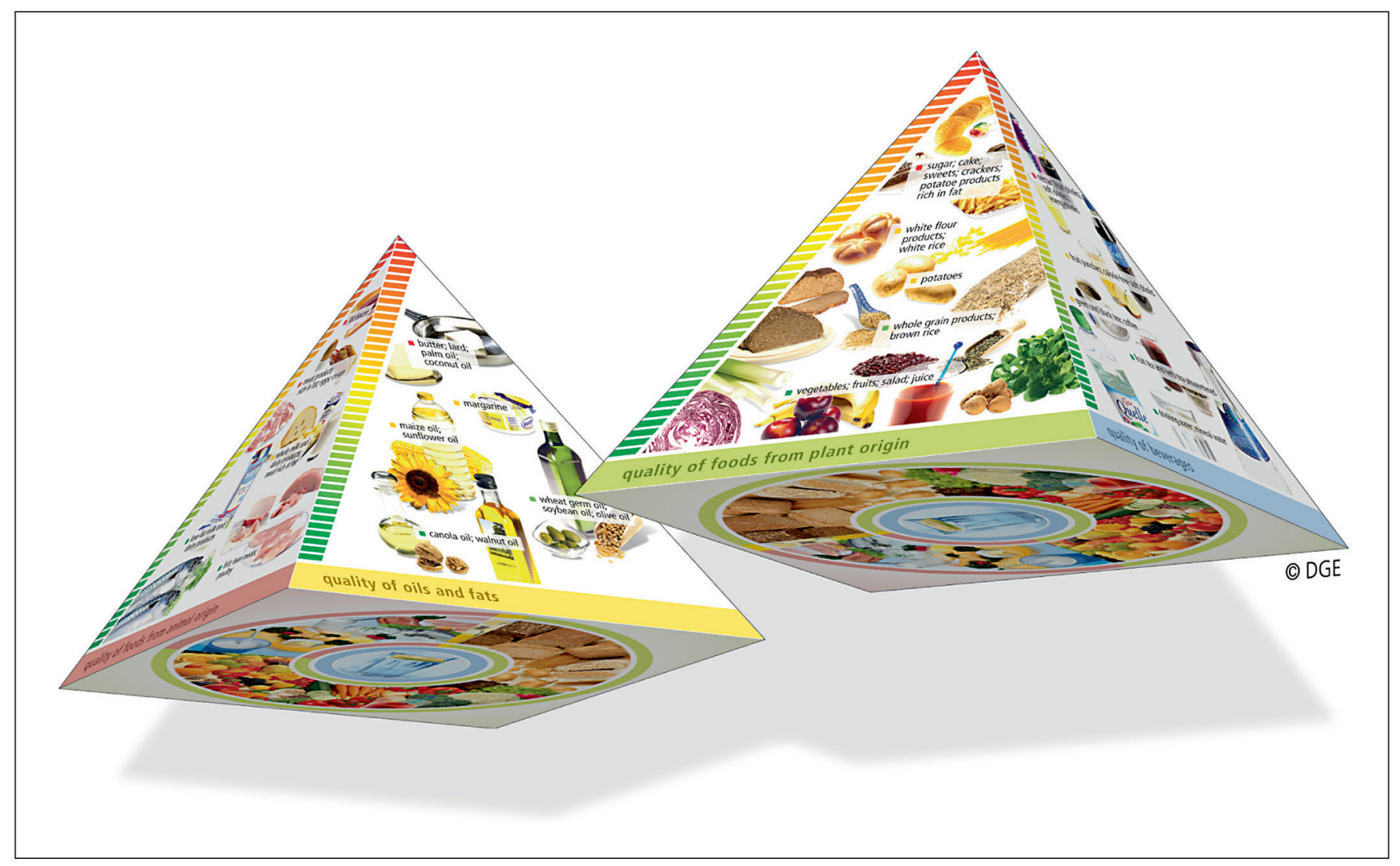

Fig. 3. The 3D Food Guide Pyramid, 2006.

Food Pyramid) (fig. 3) adding important qualitative information for an adequate food choice. Qualitative ranking of food is based on the so-called 'metabolic' approach: consideration of actual data from epidemiological studies describing metabolic and clinical effects of distinct foods and/or food classes [2].

The square base of the 3D Food Pyramid represents the Nutrition Circle (quantitative approach; see above) but condensed to four food classes. The four sloping sides are set to qualitatively rank products (shown as pictures/ icons). On the basis of selected criteria, foods were placed on the bottom (green: preferential use), in the middle (yellow: use frequently) and on the top (red: use sparingly) of the triangle.

Foods of mainly plant origin (including cereals, bread and pastries): Major nutritive criteria for the qualitative ranking were energy density and the presence of physiologically valuable substrates like phytochemicals and dietary fiber. The glycemic index was only of minor importance. Preventive effects of distinct foods on the prev- alence of chronic diseases were additionally considered. At the bottom of this side and, thus, to be consumed in larger quantities, fruits and vegetables are placed (generally low caloric, high in bioactive substrates). The second level is reserved for full bread, potatoes and low fat potato products, rice and white bread. Sweets and energyrich snacks are at the top and should be consumed sparingly.

Foods of mainly animal origin (including meat, milk and fish products): The nutritional quality within this food class is assessed on the basis of energy density, fat content, fatty acid composition (content of essential fatty acids) and nutrient density with respect to calcium, iron, zinc, selenium, and vitamins $\mathrm{B}_{1}, \mathrm{~B}_{2}, \mathrm{~B}_{6}, \mathrm{~B}_{12}$, and $\mathrm{D}$. Similar to the evaluation of plant-derived food, preventive aspects from epidemiological studies with respect to chronic degenerative diseases were taken into account. The broad basis of the triangle is formed by fish, low-fat milk products, lean red and white meat. With decreasing physiological quality, foods like full-fat milk products/cheese, 
fat meat and meat products, eggs and bacon are ranked at the top.

Fats and oils: In addition to the fats delivered by foods of plant and animal origin, this food group should provide only $3 \%$ of daily total energy consumption. Decisive criteria for the qualitative assessment were the fatty acid composition (saturated, monounsaturated and polyunsaturated fatty acids; trans-fatty acids; ratio n-6:n-3) and the ratio of vitamin $\mathrm{E}$ to the number of double bonds present. In addition, practical aspects (use of oils/fats in cooking) as well as preventive aspects have been considered. Plant oils from canola, olives, soy, walnuts should be preferably chosen, followed by maize and sunflower oil.

Beverages: Assessment of quality was mainly based on energy density (\% carbohydrates/l), content of bioactive substrates, glycemic index and caffeine content. At the bottom energy-free beverages (tap/mineral water, sugarfree fruit and herbal teas: consumption not limited) are placed. The yellow color was given to beverages with a carbohydrate concentration $<70 \mathrm{~g} / \mathrm{l}$ ( $7 \%$; tea/coffee with added sugar, juices diluted with water, alcohol-free beer). The top (use sparingly) of the triangle is occupied by high-calorie beverages like so-called energy drinks and soft drinks. Alcoholic beverages are not considered due to the known risks for abuse.
First tests with target groups (health professionals, teachers, young consumers) showed a high rate of acceptance and demonstrated successful use of this new model in an interactive mode. In addition to computer applications and animations, two-dimensional versions ('nutrition compass') are produced as paper prints and folders.

\section{Summary and Conclusions}

In Germany, nutritional knowledge is successfully disseminated using different validated instruments which are based on actual scientific evidence. Quantitative aspects can be easily enlightened with the help of the Nutrition Circle. That lifestyle is an important factor to stay healthy is transported using a flyer wording 10 Nutrition Guidelines. The 3D Food Guide Pyramid is an encouraging new instrument to establish new teaching programs with a high level of acceptance. Within the concept it is planned to establish target-specific food guide pyramids, e.g. for adolescents, aged persons (considering gender), people with increased physical activity and others. Using modern communication technology, it will be feasible for the consumer to download an individual pyramid considering age, gender, physiological and socio-economic situation.

\section{References}

1 German Nutrition Society, Austrian Nutrition Society, Swiss Society for Nutrition Research, Swiss Nutrition Association: Reference Values for Nutrient Intake, ed 1. Frankfurt, Umschau Braus Publisher GmbH, 2002.

2 Stehle P, Oberritter $\mathrm{H}$, Buening-Fesel M, Heseker $\mathrm{H}$ : Graphics illustrating nutritional guidelines - traditional and new approaches (in German). Ernähr Umsch 2005;52:146148.
-3 Choumenkovitch SF, Selhub J, Wilson PWF: Folic acid intake from fortification in United States exceeds predictions. J Nutr 2002;132: 2792-2798.

4 Krawinkel M, Broenstrup A, Bechthold A, Biesalski HK, Boeing H, Elmadfa I, Heseker $\mathrm{H}$, Kroke A, Leschik-Bonnet E, Oberritter $\mathrm{H}$, Stehle P: Strategies to improve folate supply in Germany - risks and benefits. Position paper of the German Nutrition Society (in German). Ernähr Umsch 2006;53:424-429.
5 Anonymous: Examples of foods in current sets of dietary guidelines and food guides; in FAO (ed): Preparation and Use of FoodBased Dietary Guidelines. Report of a Joint FAO/WHO Consultation, Nicosia, Cyprus, 1996.

6 Painter J, Rah JH, Lee YK: Comparison of international food guide pictorial representations. J Am Diet Assoc 2002;102:483-489. 